\title{
Hommage à Gilbert Durand
}

\section{Norbert Germanaz}

\section{(2) OpenEdition}

\section{Journals}

\section{Édition électronique}

URL : http://journals.openedition.org/studifrancesi/2043

DOI : 10.4000/studifrancesi.2043

ISSN : 2427-5856

\section{Éditeur}

Rosenberg \& Sellier

\section{Édition imprimée}

Date de publication : 1 avril 2014

Pagination : 108-112

ISSN : 0039-2944

\section{Référence électronique}

Norbert Germanaz, « Hommage à Gilbert Durand », Studi Francesi [En ligne], 172 (LVIII | I) | 2014, mis en ligne le 01 avril 2014, consulté le 18 septembre 2020. URL : http://journals.openedition.org/ studifrancesi/2043; DOI : https://doi.org/10.4000/studifrancesi.2043

\section{(c) (i) (9)}

Studi Francesi è distribuita con Licenza Creative Commons Attribuzione - Non commerciale - Non opere derivate 4.0 Internazionale. 


\title{
Hommage à Gilbert Durand
}

\begin{abstract}
This article analyses firstly a few statements released in memory of Gilbert Durand after his death in 2012, then the meaning of his study of the imaginary as it was explained by the philosopher himself in his lecture Fondements et perspectives d'une philosophie de l'imaginaire (Québec 1989), and finally the mythocritical and mythoanalytical methods that he applied to art and literature. A connection appears clearly between Gilbert Durand's biography - his commitment to the fight against totalitarianism and the painful experiences he endured - and the direction of his research determined by the crisis of a long-established value system. Durand's research was inspired by Gaston Bachelard's teaching, while he discovered the notion of archetype, which he investigated in depth, in Jung's works; thanks to Henry Corbin, he became aware of the religious matrix in the formation of human psyche.
\end{abstract}

Un hommage a été rendu le 7 décembre 2012 par la Ministre de l'enseignement supérieur et de la recherche à Gilbert Durand, pour son engagement dans la lutte de la Résistance Française et pour son activité universitaire (professeur émérite de l'Université Stendhal à Grenoble où il fut d'abord professeur de philosophie puis de sociologie et d'anthropologie): le communiqué officiel rappelle que cet éminent universitaire est l'un des précurseurs des recherches sur l'imaginaire humain et souligne le caractère transdisciplinaire de sa méthode qui ne prend pas en considération les découpages disciplinaires, parce que ceux-ci ignorent la puissance de l'image. Une précision sur le contexte historique est également apportée: la conception théorique de l'imaginaire de G. Durand est à replacer à la fin des années cinquante, à la naissance du structuralisme, dont elle garde l'empreinte dans le goût des formes abstraites et de leur combinaison. Ainsi ce philosophe est-il l'auteur d'une quinzaine d'ouvrages dont le plus connu, Les structures anthropologiques de l'imaginaire 1 , a été l'objet d'étude d'au moins deux générations de chercheurs.

Précisément dans le Journal des chercheurs du 9 décembre 2012, René Barbier évoque deux propositions de Durand sur l'origine de l'imaginaire et sur l'organisation de son contenu. Ainsi l'origine de l'imaginaire est dans une réponse à l'angoisse existentielle et universelle liée à l'expérience négative du temps, de cette angoisse naîtrait l'imaginaire. Afin de rompre avec les réductionnismes, le philosophe grenoblois tente de cerner l'activité de l'imaginaire dans un «incessant échange entre les pulsions subjectives et assimilatrices et les intimations objectives émanant du milieu cosmique et social».

De son côté, l'association francophone internationale de recherche scientifique en éducation, soulignant l'importance de cette recherche sur l'imaginaire, cite l'ouvrage de Durand Science de l'bomme et tradition comme fondement d'une pensée 
érudite et originale - la pensée traditionnelle, en effet, n'opère pas de coupure entre multiple et un: «C'est l'unité [...] repérée dans le monde qui se réverbère dans un moi ressenti comme divers $\rangle^{2}$. C'est ce que soutiennent d'autres qui relèvent la conception unitaire du cosmos. La plupart des hommages évoquent l'importance de l'œuvre, suivant sa progression, ses perspectives, le cheminement spirituel de l'auteur, mais en associant l'homme engagé, en l'introduisant souvent au cœur même de l'analyse: sa carrière d'enseignant a commencé à Chambéry où il a créé le premier Centre de recherche sur l'imaginaire et a tenu un rôle majeur dans la création de l'Université de Savoie. C'est aussi dans cette ville qu'il a exercé son activité de résistant et où il a été arrêté et torturé par la Gestapo dont il est resté prisonnier plusieurs mois avant d'être envoyé au camp de Compiègne pour être déporté en Allemagne mais il a été libéré par les Forces Françaises de l'intérieur. Ce combat s'inscrit dans un cheminement cohérent de lutte contre les logiques totalisantes. Mais G. Durand fut aussi nommé «le philosophe grenoblois» car la plus grande partie de sa carrière universitaire s'est déroulée à Grenoble.

Laissons-lui la parole à travers le compte-rendu d'une conférence situé au point d'arrivée d'un parcours déjà long, en 1989 à Québec (Université du Québec, Département des Sciences religieuses, $57^{\mathrm{e}}$ congrès de l'association francophone) $)^{3}$. L'auteur précise d'ailleurs l'extension un peu vague de cette philosophie, qui repose sur le seul imaginaire qui nous soit connaturel et accessible, celui de l'Homo sapiens. Il évoque le jeune homme en colère qu'il était au sortir de la guerre, devant la faillite générale des systèmes de valeurs et rappelle sa rencontre avec l'éminent maître Gaston Bachelard qui lui révèle le rapprochement entre la pensée scientifique et les autres formes de pensée: il n'y a plus opposition radicale entre sciences exactes et procédés de l'imaginaire. C'est donc Bachelard qui impulsa la recherche de Durand. Celui-ci essayait de définir d'une façon objective la subjectivité constitutive de l'Homo sapiens et l'auteur dévoile, avec humour, le sous-titre follement ambitieux de sa recherche: introduction à l'archétypologie générale. Au sujet du concept d'archétype, dans une pointe polémique, il dit être condamné pour délit de jungisme puisque c'est Jung qui a défendu et illustré ce concept. Et c'est dans la science de l'homme et tous ses horizons physiologiques, embryologiques, historiques, neurologiques, culturels et sociaux que l'on peut repérer les constances archétypales qui identifient et rendent possible la compréhension de l'Homo sapiens.

Une nouvelle étape s'ouvre en 1962 dans la recherche de Durand avec Henry Corbin, avec qui il fonde en 1974 le Centre des recherches spirituelles comparées à l'Université St. Jean de Jérusalem. La réflexion collégiale sur le changement allait orienter sa recherche car de son travail même, de l'établissement de son modèle, l'imaginaire, naissent des perspectives dynamiques. Il peut maintenant discerner, dans un ensemble socioculturel en devenir, de grands sous-ensembles cohérents qu'il appelle bassins sémantiques car il s'est peu à peu débarrassé de la notion de progrès pour prendre en considération la notion de cycle historico-socio-culturel et il parvient à repérer dans ces bassins sémantiques des phases qu'il nomme ruissellement, partage des eaux, confluences, aménagement des rives, méandres, deltas. Il loue à ce sujet la perspicacité de l'historien français Fernand Braudel qui dégage des rythmes différents dans une société donnée et invite le sociologue à examiner les longues durées historiques. Certes, conclut G. Durand, dans ce système dynamique,

(2) G. Durand, Science de l'homme et tradition. Le nouvel esprit anthropologique, Paris, Tête de feuilles/Sirac, 1975 , p. 37.

(3) «Fondements et perspectives d'une philoso- phie de l'Imaginaire», in Le Statut de l'Imaginaire dans l'auvre de G. Durand, «Religiologiques», n. 1, Univ. du Québec à Montréal, 1990, pp. 27-44. 
bien des questions restent posées mais les perspectives ouvertes à la recherche sont nombreuses et passionnantes. Il cite comme un acquis les belles thèses de Gilbert Bosetti ${ }^{3}$ et d'Aurore Frasson-Marin ${ }^{4}$. Avec optimisme et enthousiasme, le philosophe affirme qu'il y a encore de grandes conquêtes à réaliser par ses méthodes mythocritiques et mythanalytiques.

Dans son introduction à la mythodologie, G. Durand développe longuement ce nouveau concept de mythodologie, méthode conçue pour réactiver la lecture des mythes, et appartenant à la science des mythes. Le mythe n'est plus un fantasme gratuit que l'on subordonne au perceptif et au rationnel. C'est une res réelle que l'on peut manipuler pour le meilleur et pour le pire. Pourquoi le mythe réapparaîtil? Comment l'un des peuples qui a nourri la culture européenne a-t-il pu adhérer, jusqu'au crime, au système simpliste du Mythe du vingtième siècle (1930), ouvrage du théoricien du nazisme Alfred Rosenberg. C'est que le nazisme, comme la Révolution française, a fourni à un peuple une prothèse du religieux dont l'allemand du Kulturkampf comme le français des Lumières était sevré.

La mythocritique est une méthode de critique littéraire ou artistique qui centre le processus compréhensif sur le récit mythique inhérent à la signification de tout récit. Structures, histoire ou milieu socio-historique tout comme appareil psychique sont indissociables et fondent l'ensemble compréhensif ou significatif de l'œuvre d'art et spécialement du récit littéraire. Chaque séquence lue constitue un mythème. La mythocritique va donc d'emblée chercher l'être même de l'œuvre dans la confrontation de l'univers mythique qui forme le goût ou la compréhension du lecteur, et l'univers mythique qui émerge de la lecture de telle œuvre déterminée; d'ailleurs critique thématique et mythocritique partagent nombre de procédés: prise en compte de la totalité de l'œuvre, recherche de structures signifiantes.

G. Durand perfectionne une mythocritique appliquée à diverses œuvres littéraires d'auteurs français et étrangers du dix-neuvième siècle, parmi lesquels Stendhal, Baudelaire, Proust, Gide, Zola. La mythocritique met en évidence chez un auteur, dans une œuvre d'une époque et d'un milieu donnés, les mythes directeurs et leurs transformations significatives. L'application de la théorie à un objet littéraire est déjà manifeste dans l'œuvre de notre philosophe dès le choix de sa thèse secondaire en 1952 consacrée à un roman de Stendhal, Le décor mythique de la chartreuse de Parme (1990, Editions José Corti) avec son sous-titre: les structures figuratives du roman stendhalien.

Dans un essai sur «La création littéraire» dans Encyclopedia Universalis ${ }^{4}$ («Spécificité des œuvres d'art») l'auteur évoque un milieu social qu'elle informe. Cette création sociale peut être formulée par une théorie de la réception selon laquelle c'est bien l'œuvre qui crée la sensibilité d'un groupe, contrairement à l'hypothèse réductrice inverse. Don Quichotte féconde l'âme espagnole du quichottisme. Dans cette analyse, point d'application de son esthétique structurale, Durand relie le romanesque du dix-neuvième siècle aux grands mythes de l'Antiquité classique. Il insiste sur le mélange du réel et de l'imaginaires. L'expérience artistique n'instaure pas une coupure entre le concept de réalité et celui d'imaginaire: il y a en effet un travail sur le réel à travers les fantasmes et les pulsions propres au sujet.

De la mythocritique l'on passe naturellement, par glissement, selon l'expression du philosophe, à la mythoanalyse: c'est le passage du texte littéraire à tous les contextes qui le baignent ${ }^{6}$. Si la mythocritique est un terme réservé à l'analyse littéraire

(4) Le mythe de l'enfance dans le roman italien contemporain, Grenoble, Ellug, 1987.

(5) Italo Calvino et l'imaginaire, Genève, Slatkine, 1986.
(6) «Les fondements de la création littéraire», in Encyclopaedia Universalis, Supplément II, Les Enjeux, 1984, p. 119-127. 
et artistique, en revanche la mythanalyse étudiera les phénomènes socioculturels, par exemple comment une période culturelle donnée, dans un lieu et un contexte historique précis, est tributaire, dans ses expressions diverses, d'un mythe ou d'une figure mythique qui s'impose peu à peu - et ensuite dégénère - parce qu'elle est confrontée à des problèmes spécifiques, certes, mais qui se sont aussi posés, sous d'autres formes en d'autres lieux à d'autres groupes humains; par exemple la place de l'homme face aux puissances supérieures, sous le signe de Prométhée dans la première moitié du dix-neuvième siècle. Ce qui montre aussi pour Simone Vierne, qu'il est pratiquement impossible de ne faire que de la mythanalyse ou que de la mythocritique car dans une perspective mythocritique, il est évident que le contexte socioculturel joue sur la création particulière et dans une perspective mythanalytique, les œuvres d'art sont partie prenante dans l'analyse des données socioculturelles où la plus grande partie des exemples pris à l'appui de ce mythe sont tirés d'œuvres littéraires. Et l'universitaire grenobloise conclut: «Le modèle durandien se présente comme une complétude qui conserve nombre de questions ouvertes. Il en va ainsi de la qualité des grandes œuvres. Nous respecterons ce choix qui permet une lecture transversale et des interprétations multiples». D'ailleurs Gilbert Durand avait pris soin de signaler aussi bien dans sa conférence au Québec que dans son Introduction à l'archétypologie générale, citée précédemment par lui-même, les points forts et les points qui font problème dans une œuvre de recherche qui couvre plus d'un quart de siècle. Dans Figures mythiques et visages de l'œuvre à l'intérieur de la rubrique «De la mythocritique à la mythanalyse»", les rapports croisés de celles-ci sont indiqués: «les visages de l'œuvre que met à jour la mythocritique, et les figures mythiques dont la mythanalyse signe une époque, se regardent et par ce regard croisé nous regardent en notre singulière modernité».

Bien avant sa disparition, le philosophe a reçu de nombreux hommages qui semblent annoncer les hommages postérieurs et se mêler à eux. Ainsi, à l'occasion de la publication de Champs de l'imaginaire en 1996, est posée par Danièle Chauvin ${ }^{10}$ cette sympathique question: «Faut-il faire oublier l'homme derrière le système?», ce contestataire, au sens noble qu'il fut toujours récusant la bassesse par son engagement dans la Résistance, récusant le conformisme intellectuel qui, à travers tant de modes, d'un marxisme étroit ou d'un structuralisme dur et une psychanalyse réductrice, entasse les théories... Faut-il enlever le charme du primesaut, cette séduction d'une spontanéité qui trouve sa rigueur dans l'enchaînement organique des étapes de la découverte, les notions s'impliquant les unes dans les autres dans une croissance toute naturelle. De son côté, la motivation première des rencontres de Nice en 2001 est d'esquisser une reconnaissance collective à Gilbert Durand venu faire partager son immense savoir, son acuité intellectuelle, sa générosité. Citons enfin une appréciation laudative en apparence plus restrictive de Jérôme Souty en 2006. Celui-ci observe que Durand a parfois fait l'objet de vives critiques en France, l'orthodoxie universitaire le percevant longtemps comme un trouble-fête, mais que son œuvre est largement diffusée et admirée à l'échelle internationale. L'œuvre de Durand est en effet une œuvre en marge car ayant le mérite d'avoir commencé à dégager un point de vue synthétique sur l'imaginaire, Durand est, de ce fait, un franc-tireur et donc en marge

(7) Voir M. Rami, L'imaginaire, publié dans le site www.e-litterature.net (Exigence - Littérature), 25 mars 2010.

(8) «C'est bien Gilbert Durand qui a mis au point et expérimenté la méthode d'analyse qui se fonde sur le mythe», S. VIERNE, Mythocritique et mythanalyse, «Iris», n. 13, 1993, pp. 43-56.
(9) G. Durand, Figures mythiques et visages de l'œuvre: de la mythocritique à la mythanalyse, Paris, Berg international, 1979.

(10) Champs de l'imaginaire, textes de G. DuRAND réunis par D. CHAUvin, Grenoble, Université Stendhal - Ellug, 1996. 
de l'Université. Il est à replacer dans la lignée de chercheurs originaux et fructueux comme Gaston Bachelard. L'œuvre est en marge aussi parce que la plupart des ouvrages sont exigeants - parfois difficiles d'accès. L'auteur convoque de nombreuses références, développe une pensée foisonnante et qui s'exprime souvent sous la forme d'une prose baroque. En général, l'écriture est exacte, rigoureuse, notent d'autres critiques. Il n'en reste pas moins que, comme le rappelle Robert Maggiori dans sa présentation de l'Introduction à la mythodologie ${ }^{11}$, le public «n'a jamais vraiment tiré de l'ombre» Gilbert Durand.

NORBERT GERMANAZ

(11) R. MAgGioRI, «Libération» 15 février 1996; G. DuRAND, Introduction à la mythodologie, Préface 